\title{
Sistema de Laboratorios Remotos para la práctica de Ingeniería de Control
}

\author{
Remote Laboratory System for practices of Control Engineering \\ Sistema de Laboratórios Remotos para a prática de Engenharia de Controle \\ Omar Mar-Cornelio1 ${ }^{\circledR}$ Iván Santana-Ching² Jorge González-Gulín³
}

Recibido: noviembre de 2018

Aceptado: agosto de 2019

Para citar este artículo: Mar-Conelio, O.; Santana-Ching, I.; González-Gulín, J. (2019). Sistema de Laboratorios Remotos para la práctica de Ingeniería de Control. Revista Científica, 36(3), 356-366. Doi: https://doi. org/10.14483/23448350.14893

\section{Resumen}

En Cuba la formación de especialistas en automática se realiza mediante la carrera de Ingeniería en Automática, la cual tiene dentro de sus disciplinas fundamentales la de Sistemas de Control. Para el desarrollo de prácticas de laboratorios, los estudiantes trabajan en dispositivos físicos o remotos, presencial o a distancia, mediante Sistemas de Laboratorios Remoto (SLR). Sin embargo, las soluciones actuales no poseen soporte o son propietarias imposibilitando su implementación para el desarrollo de prácticas de Ingeniería de Control en el sistema educacional cubano. La presente investigación describe una solución al problema planteado a partir del desarroIlo de un SLR que permite el desarrollo de prácticas de laboratorios de Ingeniería de Control. El sistema propuesto pone en funcionamiento la identificación de sistemas, sintonía de controladores y la ejecución de prácticas en dispositivos reales. Permite, además, un conjunto de reportes sobre los fenómenos analizados en cada práctica. Se aplicó una encuesta para medir el nivel de satisfacción de los usuarios respecto a la propuesta desarrollada mediante la técnica ladov, obteniéndose un alto nivel de satisfacción.

Palabras clave: ingeniería de control, prácticas de laboratorios, laboratorios remotos.

\begin{abstract}
For the development of laboratory practices, students work in physical devices or remotely, in person or remotely using Remote Laboratory Systems (SLR). However, current solutions do not have support or are proprietary, making it impossible to implement them for the development of Control Engineering practices in the Cuban Educational System. The present investigation describes a solution to the problematic one raised from the development of a System of Remote Laboratories that allows the development of practices of laboratories of Control Engineering. The proposed system allows the identification of systems, tuning of controllers and the execution of practices in real devices. It also allows a set of reports on the phenomena analyzed in each
\end{abstract}


practice. A survey is applied to measure the level of satisfaction of the users with respect to the proposal developed through the ladov technique, obtaining a high level of satisfaction.

Keywords: control engineering, laboratory practices, remote laboratories.

\section{Resumo}

Em Cuba a formação de especialistas em automação é feita através da carreira de Engenharia de Automação que possui em suas disciplinas fundamentais, a disciplina Sistemas de Controle. Para o desenvolvimento de práticas laboratoriais, os alunos trabalham em dispositivos físicos ou remotamente, pessoalmente ou remotamente, utilizando o Remote Laboratory Systems (SLR). No entanto, as soluções atuais não têm suporte ou são proprietárias, impossibilitando sua implementação para o desenvolvimento de práticas de Engenharia de Controle no Sistema Educacional Cubano. A presente investigação descreve uma solução para a problemática levantada a partir do desenvolvimento de um SLR que permite o desenvolvimento de práticas de laboratórios de Engenharia de Controle. O sistema proposto implementa a identificação de sistemas, o ajuste de controladores e a execução de práticas em dispositivos reais. Também permite um conjunto de relatórios sobre os fenômenos analisados em cada prática. Uma pesquisa é aplicada para medir o nível de satisfação dos usuários em relação à proposta desenvolvida a través da técnica de ladov, obtendo um alto nível de satisfação.

Palavras-chaves: engenharia de controle, práticas laboratoriais, laboratórios remotos.

\section{Introducción}

A partir del desarrollo tecnológico alcanzado por la sociedad, el ser humano comenzó a mecanizar los procesos industriales con el objetivo de simplificar el trabajo que realizaba. Para ello, se elaboraron ingeniosos instrumentos y máquinas que se utilizaron como herramientas en los procesos industriales.

Sin lugar a duda, la mecanización representó un paso importante como parte del desarrollo tecnológico, pero aumentar la productividad impuso nuevos retos. A partir del desarrollo energético y la motorización de los procesos mecánicos surgen los sistemas industriales, permitiéndose la elaboración con mayor eficiencia de producciones en serie.

Los sistemas industriales, para sus operaciones, requerían mantener o estabilizar determinados parámetros en su funcionamiento, dando inicio a los sistemas de control. Los sistemas de control permiten la operación de procesos de forma automática sustituyendo los mecanismos humanos de medición y actuación.

La automatización rápidamente fue introducida en los procesos fundamentales que acompañaban al hombre. La nueva ciencia comenzó a desarrollarse para asumir las demandas de los diferentes procesos industriales que requerían modernos métodos de control.

La formación de profesionales para asumir las demandas de automatización representaba una tarea importante a cometer en el nuevo contexto tecnológico. En Cuba desde la década de 1960 con la llegada de especialistas checoslovacos se comienzan a impartir cursos relacionados con el control automático. Así mismo, en 1963 se crea en La Habana la Escuela de Automatización Industrial y a finales de esa década pasan estos cursos a ser impartidos también en la Universidad de Oriente (UO) y la Universidad Central Marta Abreu de las Villas (UCLV).

En la década de 1980 evoluciona hacia la Ingeniería en Automática pasando por transformaciones de los planes de estudios hasta la actualidad que es impartida en la UO, en el Instituto Superior Politécnico José Antonio Echeverría, actualmente Universidad Tecnológica de La Habana (CUJAE) y en la UCLV. La carrera de Ingeniería en Automática define como objeto de la profesión los sistemas de control, constituidos por el proceso a controlar, los medios técnicos necesarios para realizar la medición, la supervisión y el control en su esfera de actuación (Mes, 2017; Valencia, 2016). 
Dentro de las disciplinas fundamentales de la carrera de Ingeniería en Automática para respaldar el objeto de la profesión se encuentra la de sistemas de control. Esta se compone por un grupo de asignaturas que conforman el currículo base como son: Modelado y simulación, Maquinaria eléctrica, Ingeniería de control I, Ingeniería de control II, Procesos, Control de procesos I y Accionamiento eléctrico.

Para aplicar los contenidos de la asignatura Ingeniería de control II, la misma cuenta con prácticas de laboratorios como tipología de clases. En ellos los estudiantes expresan los conocimientos adquiridos en laboratorios equipados en correspondencia con el perfil que se requiera (Saenz et al., 2015; Samuelsen y Graven, 2016). En este contexto el usuario puede realizar las prácticas en laboratorios físicos, con equipos afines a la materia recibida. Sin embargo, la solución antes expuesta representa una variante altamente costosa para ser puesta en funcionamiento.

Una alternativa al problema planteado consistiría en realizar prácticas a distancias. Para ello los estudiantes acceden a equipamientos físicos centralizados de manera remota con el empleo de laboratorios.

Un sistema de laboratorios remoto (SLR) o a distancia representa la forma de operar y controlar de manera remota sistemas reales mediante una interfaz de experimentación que se ejecuta en un ordenador conectado a una red. Esto se conoce como tele laboratorio, laboratorio remoto o tele operación a través de la web (Garay, 2015; Salas y Cerón, 2014; Sancristobal et al., 2014).

A partir del estudio de la literatura científica sobre los SLR para las prácticas de Ingeniería de control, se pudo evidenciar que las soluciones existentes en su mayoría no poseen soporte técnico o representa productos propietarios por lo que deben ser paga sus licencias para el uso y no pueden ser adecuadas a las necesidades de las instituciones cubanas. Sobre los elementos antes expuestos, se propone como objetivo de la presente investigación poner en marcha un SLR para las prácticas de Ingeniería de control.

\section{Materiales y métodos}

En la presente sesión se describen las características del SLR para las prácticas de Ingeniería de control. Se representa la arquitectura del sistema, los requerimientos de hardware y los principales elementos que facilitan la compresión de la propuesta.

\section{Características del SLR}

Los SLR son laboratorios convencionales de equipos generalmente sofisticados o exclusivos que, por medio de interfaces web, permiten la manipulación del equipo de forma remota. Este tipo de acceso facilita compartir los recursos entre varias instituciones; de esta forma, la inversión para equipamiento puede compartirse entre varias instituciones y el número de usuarios especializados se incrementa, aumentando así los beneficios académicos (Cáceres y Amaya, 2016; Jin-Hsien y Jongyun, 2007; Santana et al., 2013).

Diversas han sido las investigaciones realizadas sobre los laboratorios remotos para la enseñanza de la Ingeniería de control (Aguilar y Heredia,2013; Buitrago-Molina et al., 2014; Mar et al., 2016; Rubio et al., 2016; Sartorius et al., 2005). En las mismas se pudo constatar que cada sistema posee una arquitectura y flujo de trabajo definido en dependencia del fenómeno que se modela. Para el contexto de la presente investigación se asume la arquitectura propuesta por Ching. La figura 1 muestra la arquitectura implementada (Santana, 2012).

\section{Descripción de los componentes de la arquitectura}

En el sistema propuesto son los estudiantes (clientes web) los usuarios finales que realizan las prácticas de laboratorios de Control automático, los cuales acceden al servidor de aplicaciones mediante una conexión a la red universitaria o internet.

El servidor de aplicaciones web es el encargado de la comunicación entre los clientes web y las 


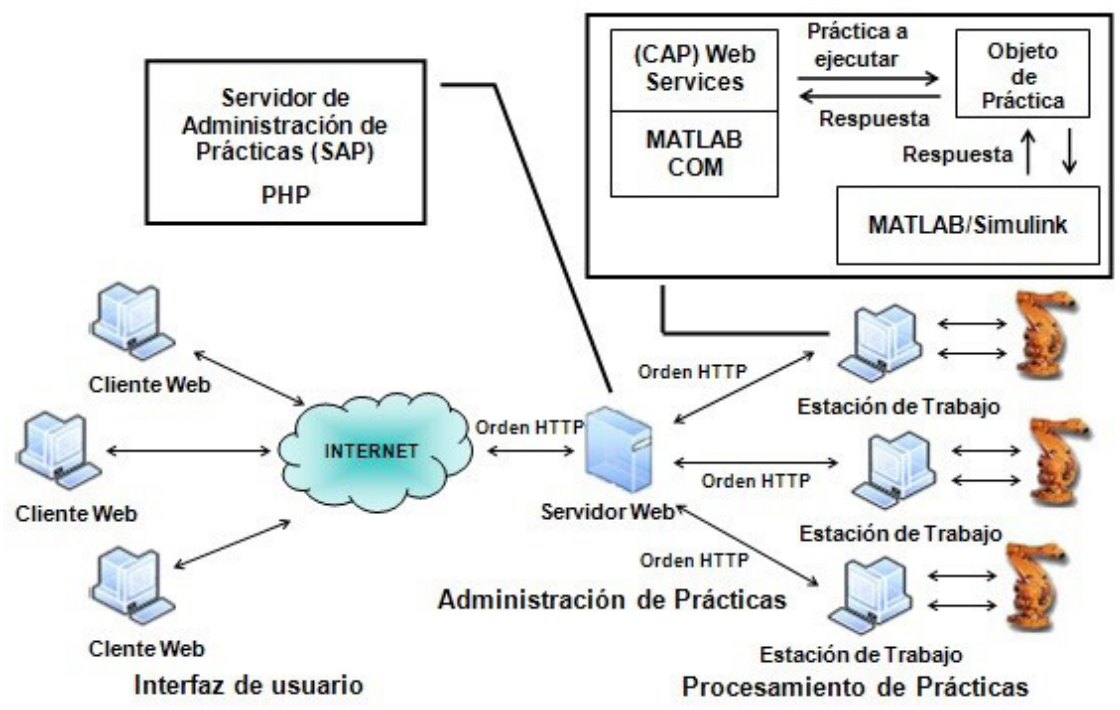

Figura 1. Arquitectura implementada en el SLR.

estaciones de trabajo, desde este se realiza la administración de las prácticas.

El servidor de administración de prácticas permite el intercambio de información mediante servicios web con las estaciones de trabajo.

Las estaciones de trabajo se conectan físicamente con las plantas que funcionaran como maqueta en el sistema. Las estaciones poseen una cámara acoplada para observar al instante el funcionamiento de sus dispositivos.

\section{Requisitos de hardware para la implementación del SLR}

Como requerimientos de hardware para garantizar el correcto funcionamiento del sistema se necesitan para las estaciones clientes del sistema una tarjeta de red de $10 \mathrm{Mb}$ o superior y estar conectadas a la LAN universitaria, una memoria operativa de $256 \mathrm{Mb}$ o superior, un procesador de $1 \mathrm{Ghz}$ o superior. Por su parte, como requisitos mínimos el servidor debe tener un microprocesador Pentium IV, una memoria operativa de $2.0 \mathrm{~Gb}$, una capacidad de almacenamiento de disco de 80 Gb y una tarjeta de red de $100 \mathrm{Mb}$.

Para la implementación de las prácticas remotas se diseñó una maqueta que gestiona el control del proceso mediante una tarjeta microcontroladora Arduino (Badamasi, 2014) como plataforma de desarrollo open hardware (Matijevic y Cvjetkovic, 2016; Murthy et al., 2014), tal como muestra la figura 2.

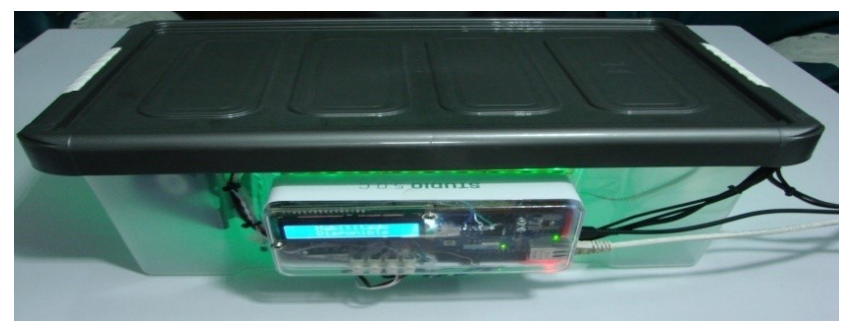

Figura 2. Maqueta para el desarrollo de prácticas de laboratorio de un sistema térmico a escala.

La maqueta representa un proceso térmico a escala, está compuesta por una armadura de polietileno en la que se estudia el comportamiento de la variable temperatura. Posee un elemento resistor en su interior encargado de disipar energía térmica la cual es re-circulada mediante un sistema de ventilación interna. Utiliza un sensor de temperatura LM35 y una tarjeta microcontroladora Arduino con una interfaz Ethernet para garantizar la comunicación con el SLR. La tarjeta microcontroladora posee la programación del controlador PID que recibe sus parámetros de ajuste del SLR y devuelve el comportamiento del proceso. 


\section{Resultados y discusión}

El SLR está orientado a soportar la gestión sobre las prácticas de laboratorios de Ingeniería de control. Está compuesto por tres funcionalidades básicas: identificación de sistema, sintonía de controladores y ejecución de prácticas en dispositivos físicos.

El flujo de trabajo permite el desarrollo de las prácticas de laboratorios a partir de una secuencia de actividades. La figura 3 muestra el flujo de trabajo para el componente.

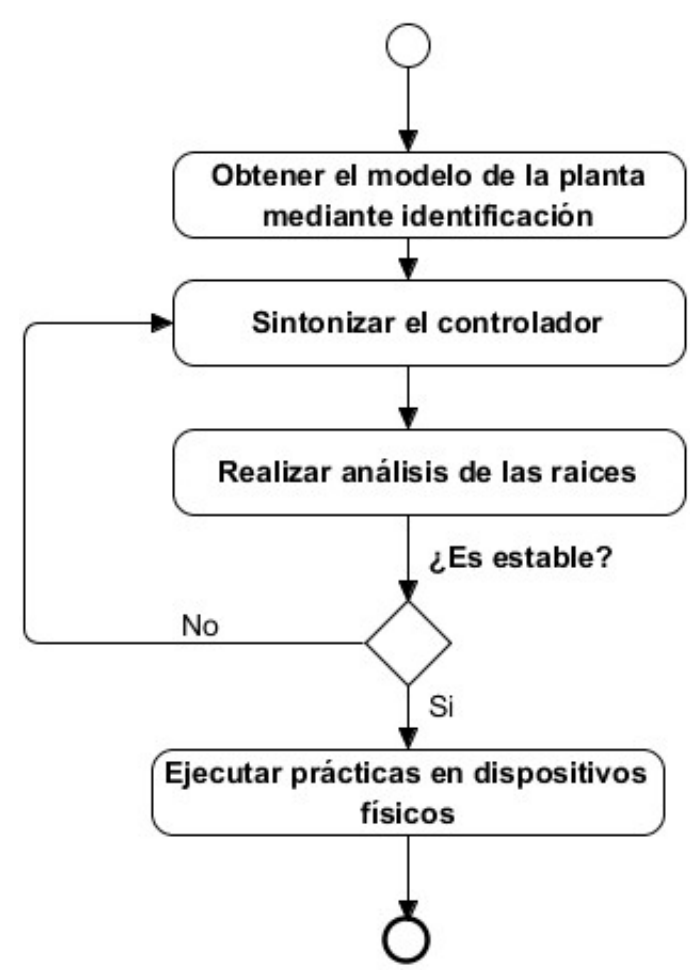

Figura 3. Flujo de trabajo para el componente Ingeniería de control.

A continuación, se presentan los resultados obtenidos para las actividades propuestas.

La identificación del sistema: permite la realización de prácticas de identificación del sistema a controlar realizándose una aproximación mediante un sistema de primer orden con retardo de transporte (PORT); posteriormente, se realiza la sintonía del controlador y se obtiene el modelo de la planta y el controlador antes de ejecutarse en los sistemas físicos, tal como muestra la figura 4.
La actividad identificar el sistema consiste en modelar la dinámica del sistema mediante la aplicación de estímulos en su entrada. Entre estos métodos se encuentra la aproximación de la respuesta del sistema cuando se aplica un estímulo de tipo escalón. La aproximación da como resultado un sistema de PORT.

El sistema PORT es caracterizado por tres parámetros: ganancia $(K)$, constante de tiempo $(\tau)$ y tiempo de retardo $(T d)$. Para el contexto de la presente investigación se propone la modelación mediante un sistema PORT tal como establece la ecuación 1.

$$
G_{(s)}=\frac{K e^{-t d s}}{\tau \mathrm{s}+1}
$$

La actividad consiste en aplicar un escalón y a partir de la curva de reacción del sistema, obtener la ganancia $(K)$, constante de tiempo $(\tau)$ y tiempo de retardo $(T d)$ que modelan un sistema PORT.

Para el proceso de identificación del sistema el estudiante debe seleccionar un escalón que será aplicado a la planta para obtener la curva de reacción. A partir de la curva de reacción el estudiante debe ser capaz de obtener los valores $(k),(\tau),(T d)$. En la actividad el estudiante obtuvo como resultado la aproximación del modelo de la planta identificada mediante PORT en dominio continuo.

La sintonía de controladores permite una vez realizada la identificación de la planta elegir la técnica para la sintonía del controlador. Primero se comienza aplicando alguna técnica empírica de parámetros del controlador como las reglas de Ziegler-Nichols. Posteriormente, se realiza una sintonización fina para mejorar sus prestaciones.

Para la sintonía del controlador es importante conocer las reglas del diseño, por ejemplo:

- Si se desea un error en estado estable igual a cero, regla que introduce el componente integrador.

- Si el tiempo de establecimiento debe ser el menor posible, regla que introduce el componente derivativo. 


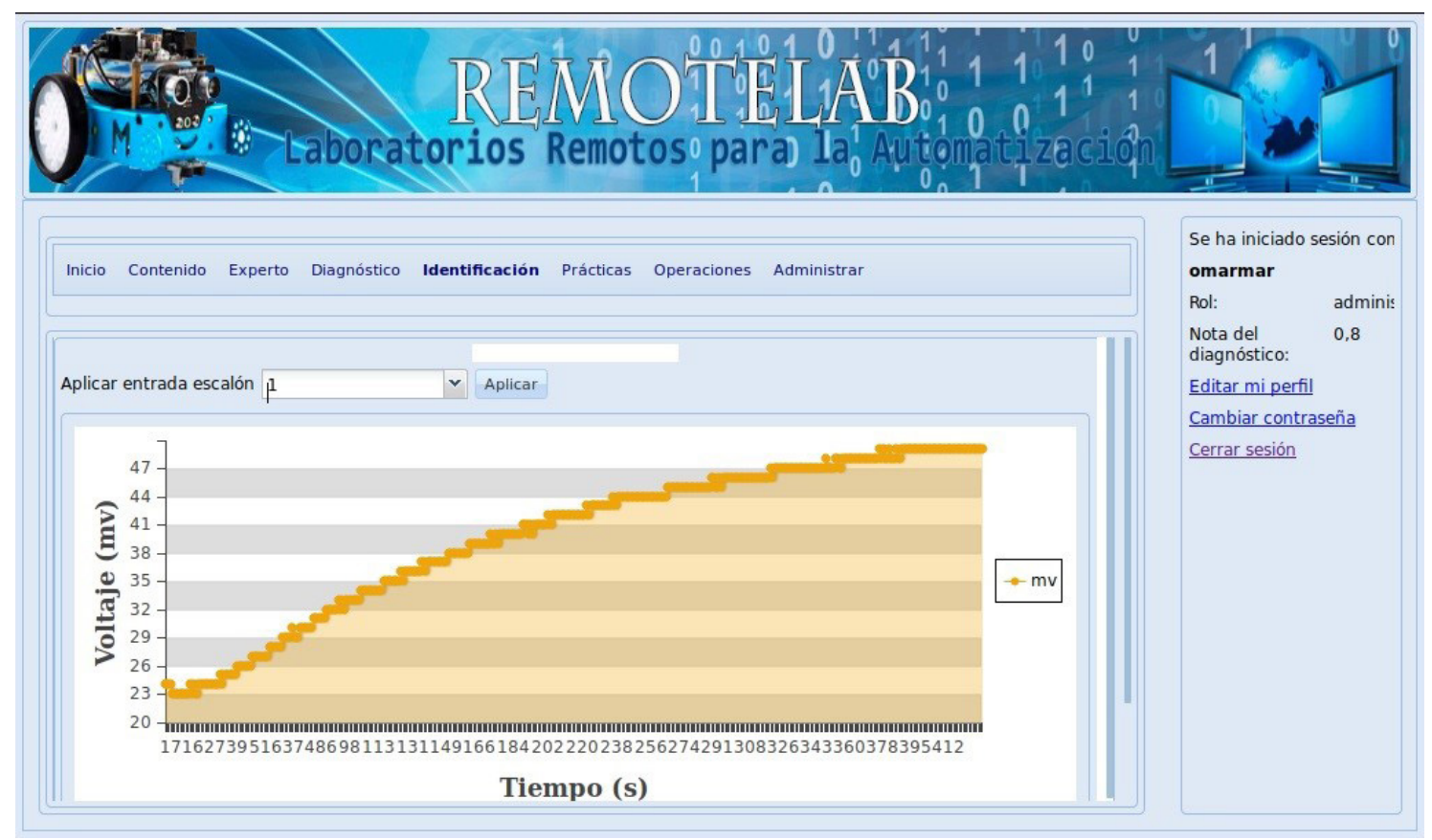

Figura 4. Interfaz en la que se ejecuta una práctica de Ingeniería de identificación y ajuste de controladores.

A partir de las reglas del diseño se decide si el controlador es P, PI o PID para que el tipo del sistema sea uno. Por Ziegler-Nichols los parámetros del controlador se calculan tal como expresa la tabla 1.

Tabla 1. Valores de los parámetros del controlador.

\begin{tabular}{|c|c|c|c|}
\hline & $K_{p}$ & $\tau_{i}$ & $\tau_{d}$ \\
\hline$P$ & $\frac{T}{L}$ & $\infty$ & 0 \\
\hline$P I$ & $0.9 \frac{T}{L}$ & $\frac{L}{0.3}$ & 0 \\
\hline$P I D$ & $1.2 \frac{T}{L}$ & $2 L$ & $0.5 L$ \\
\hline
\end{tabular}

Fuente: Ziegler y Nichols (1942).

Para obtener $L$ y $T$ se traza una recta tangente al punto de inflexión de la respuesta, la intersección con el eje del tiempo y con el valor final de la amplitud forman las distancias $L$ y $T$.

Con $L$ y $T$ se obtienen los parámetros del controlador PID obteniendo el modelo del controlador tal como muestra la ecuación 2 en dominio continuo.

$$
G_{c}(s)=K_{p}\left(1+\frac{1}{\tau_{i} s}+\tau_{d} s\right)
$$

La figura 6 muestra un formulario en el cual los estudiantes, a partir de la aplicación del método de diseño, introducen los parámetros de sintonía del controlador a utilizar en la práctica real.

Una vez obtenidos los parámetros de sintonía del controlador se realizan análisis de estabilidad. El análisis de estabilidad permite identificar el mal funcionamiento en las estaciones disponibles para el SLR (Arántegui, 2011).

El autor puso en funcionamiento un algoritmo para realizar el análisis de estabilidad a partir del análisis del lugar geométrico de las raíces utilizando funciones de Matlab. La figura 6 describe el algoritmo propuesto.

A continuación, Se describe el flujo de actividades para realizar el análisis de estabilidad:

- Obtener el modelo de la planta: a partir del proceso de identificación de la planta se obtuvo un modelo aproximado del proceso en 


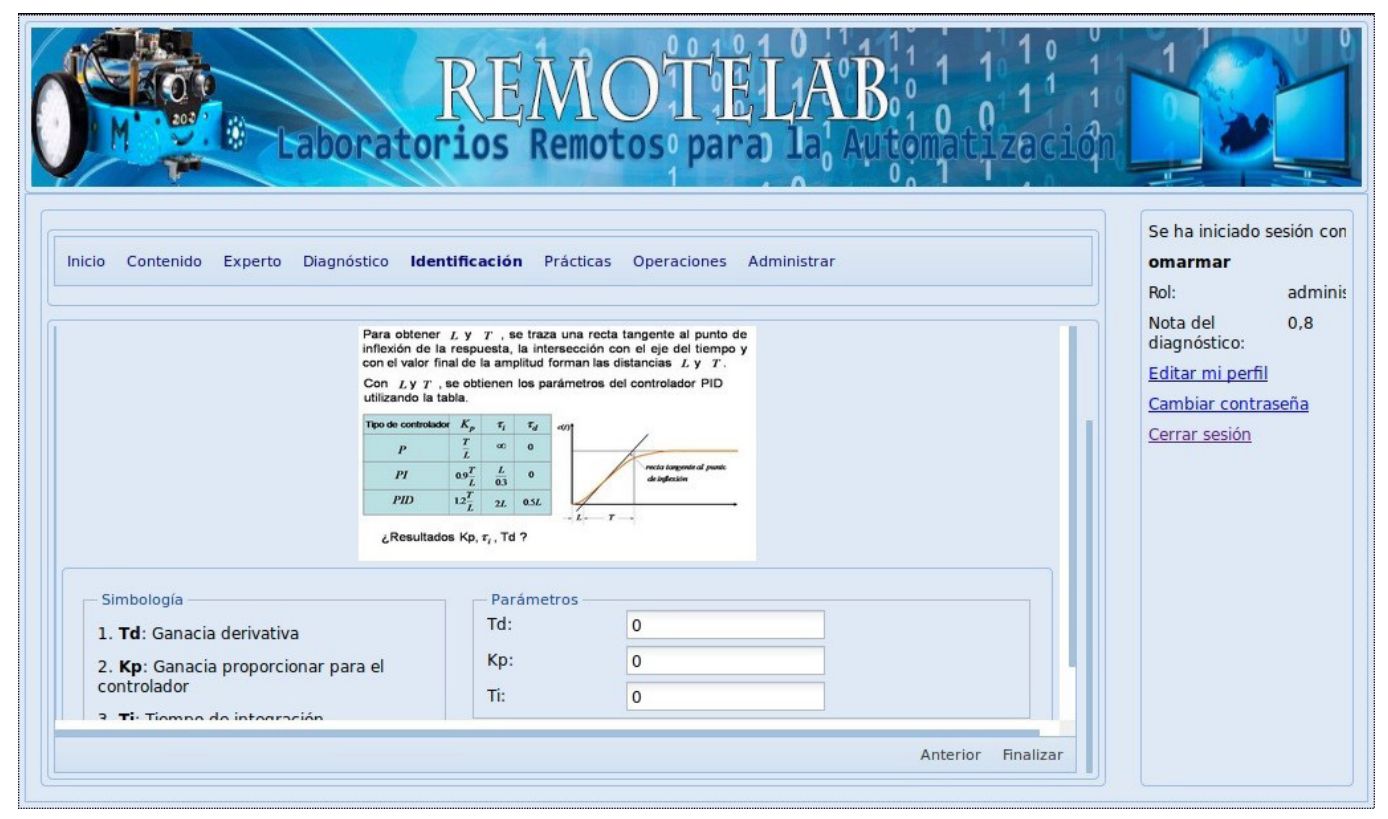

Figura 5. Interfaz en la que se realiza la sintonía del controlador.

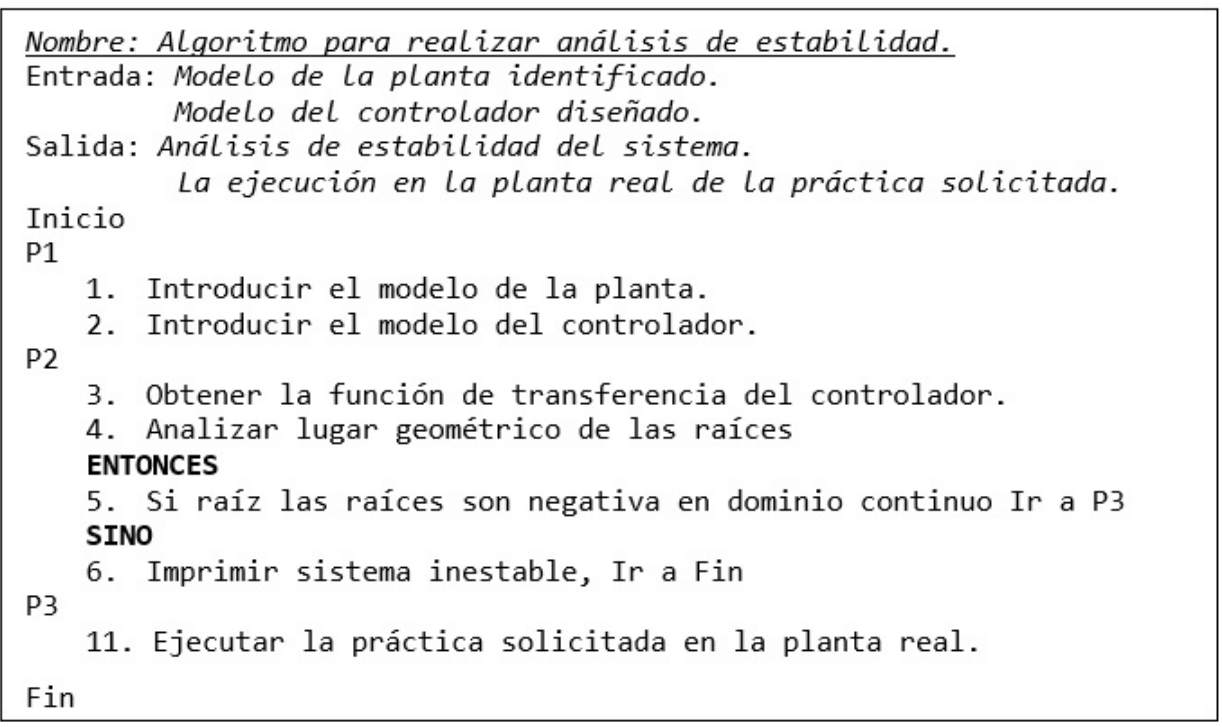

Figura 6. Algoritmo para realizar análisis de estabilidad.

la actividad anterior y es tomado como dato de entrada para el análisis de estabilidad del sistema.

- Obtener el modelo del controlador: a partir del proceso de sintonía del controlador se obtuvo el modelo del controlador que representa una entrada para el análisis de estabilidad del sistema.
- Análisis de las raíces: un sistema es estable si responde con una variación finita a variaciones finitas de sus señales de entrada. Si se considera un sistema lineal e invariante en el tiempo, la inestabilidad del sistema supondrá una respuesta que aumenta o disminuye de forma exponencial, o una oscilación cuya amplitud aumenta exponencialmente (Ogata, 
2010). Para el análisis de estabilidad se aplicó el lugar geométrico de las raíces (LGR) y se utilizó una instancia de Matlab en función de obtener el resultado (Mar et al., 2017; González, 2013).

- Ejecución de las prácticas reales: permite a partir de la sintonía de los parámetros del controlador ejecutar la práctica en el dispositivo físico. Para ello se determina la estabilidad del controlador propuesto previamente. La figura 7 muestra una vista para introducir los parámetros sintonizados del controlador. El sistema devuelve la respuesta del comportamiento de la acción de control sobre el proceso.

\section{Implementación de la técnica ladov para medir la satisfacción de los usuarios}

En investigaciones en las que el resultado final impacta sobre un conjunto de personas, la valoración para medir la satisfacción del usuario final representa una vía para validar empíricamente la propuesta presentada. La técnica ladov constituye una forma de medir el grado de satisfacción (Kuzmina, 1970).

La técnica se basa en la aplicación de una encuesta compuesta por preguntas cerradas y abiertas (Castillo y Ginoris, 2005). Las tres preguntas cerradas establecen una relación en el cuadro lógico de ladov (López y González, 2002), indicando la escala de satisfacción individual de cada encuestado; mientras que las preguntas abiertas permiten profundizar en los elementos positivos y las recomendaciones o insuficiencias de la propuesta que se evalúa.

Para la implementación de la técnica se aplicó un cuestionario a un grupo de estudiantes después de interactuar con la herramienta SLR donde realizaron prácticas de identificación de sistemas, sintonía de controladores y, por último, ejecución de prácticas en dispositivos físicos. El objetivo del instrumento realizado estuvo en función de validar la satisfacción de los estudiantes como usuarios finales.

La muestra utilizada para el desarrollo de la actividad estuvo compuesta por 28 estudiantes de la Universidad Central Marta Abreu de las Villas. Los estudiantes representan el 73,68 \% de la matrícula de cuarto año que recibe la asignatura Ingeniería de control II. Las variables evaluadas fueron las siguientes:

- Satisfacción del usuario: si el colectivo de estudiantes considera que la herramienta SLR implementada apoya las prácticas de laboratorios de Ingeniería de control II.

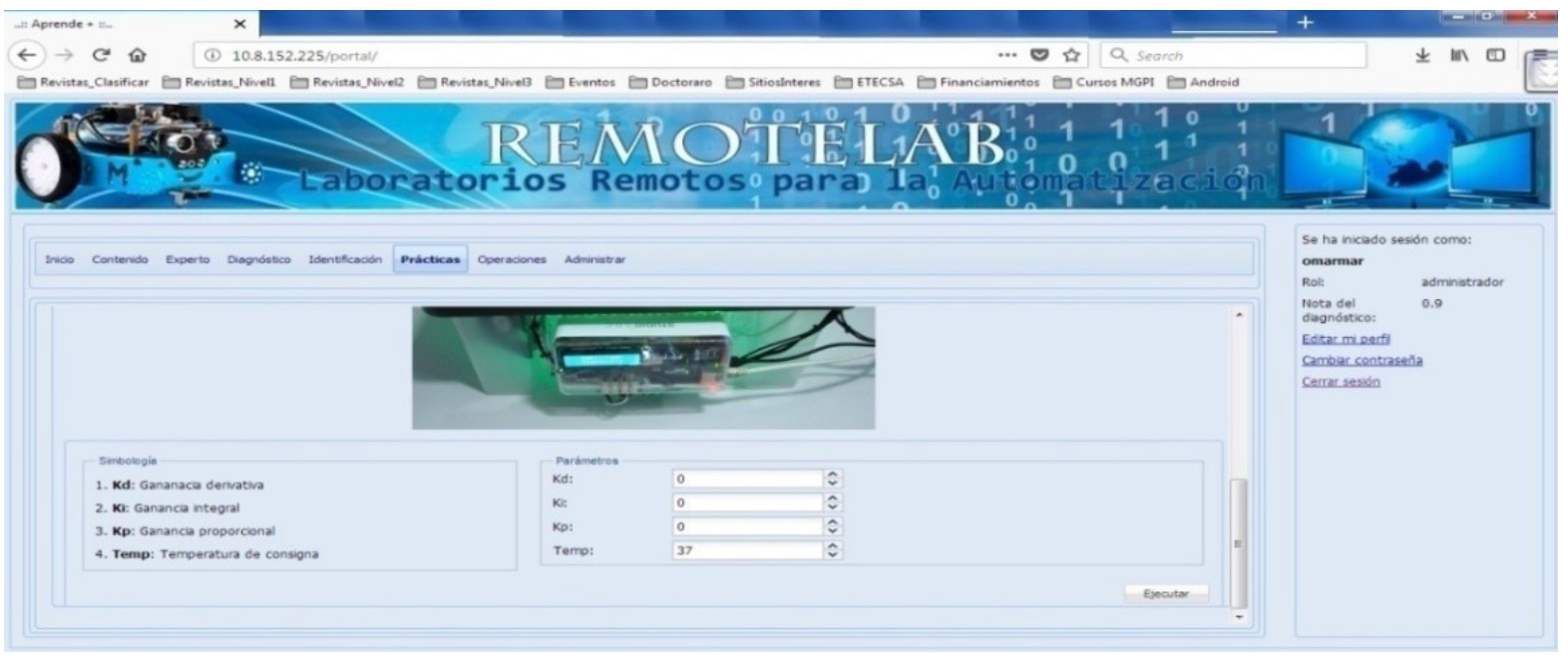

Figura 7. Interfaz en la que se ejecuta una práctica de laboratorio en el dispositivo físico. 
- Aplicabilidad de la herramienta: si el colectivo de estudiantes considera que la concepción de la herramienta es aplicable en el Sistema Educacional Cubano y representa una forma novedosa de aplicar los contenidos recibidos.

- Utilidad de la herramienta: si el colectivo de estudiantes considera que la herramienta aplica un método de inferencia para el control de acceso de las prácticas de laboratorio de Ingeniería de control II que contribuye a la auto preparación de los estudiantes para enfrentar el desarrollo exitoso de las mismas.

A partir de la aplicación de la técnica se obtiene como resultado el índice de satisfacción grupal (ISG), que representa un parámetro atribuido a la concordancia del grupo de usuarios a los que se les aplicó el instrumento. El ISG se determina mediante la ecuación 3.

$I S G=\frac{A(+1)+B(0.5)+C(0)-D(0.5)+E(-1)}{N}$

Donde: $A$ representa el número de sujetos con índice individual $1 . B$ representa el número de usuarios con índice individual 2. $C$ representa el número de usuarios con índice individual ( 3 o 6). $D$ representa el número de usuarios con índice individual 4. $E$ representa el número de usuarios con índice individual 5. $N$ : representa el número total de usuarios del grupo.

El ISG arroja valores comprendidos entre +1 y - 1 tal como se muestra en la figura 8 (López y González, 2002). Los valores comprendidos entre - 1 y $-0,5$ indican insatisfacción, los valores comprendidos entre $-0,49$ y 0,49 evidencian contradicciones por lo que se expresa como insatisfacción y los valores comprendidos entre 0,5 y 1 indican que existe satisfacción.

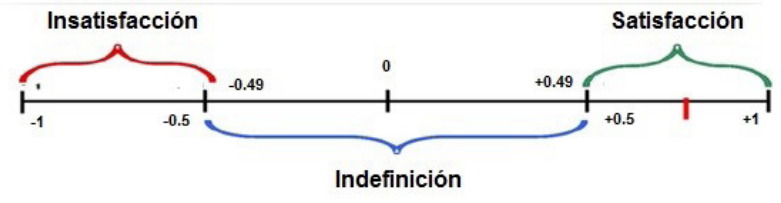

Figura 8. Índice de satisfacción.
En el proceso realizado se obtuvo un ISG = 0,892, por lo que se considera que existe alta satisfacción. A partir de obtener un ISG satisfactorio, se realiza el siguiente análisis:

- El criterio de los encuestados respecto a la pregunta "¿cuál es su criterio sobre la SLR?", se obtuvo una máxima satisfacción de 85,71 \% y el $7,14 \%$ lo consideró más satisfecho que insatisfecho y el 7,14 \% lo consideró no definido.

- Respecto a la pregunta " ¿considera usted oportuno que un SLR tenga incorporado un modelo para la toma de decisiones sobre el control de acceso a las prácticas de laboratorios a partir del diagnóstico de las habilidades?", se obtuvo una máxima satisfacción de $85,71 \%$ y el $7,14 \%$ lo consideró más satisfecho que insatisfecho; solo el 7,14 \% lo consideró no definido.

- En cuanto a si la pregunta "¿utilizaría usted la herramienta propuesta?", el 85,71\% se considera con la máxima satisfacción y el 14,28 \% se califica más satisfecho que insatisfecho.

\section{Conclusiones}

La utilización del SLR en la enseñanza de la Ingeniería de control para el desarrollo de prácticas de laboratorios de forma remota permitió que los estudiantes experimentaran sobre los contenidos teóricos recibidos en clases en dispositivos físicos.

A partir de la utilización del SLR se logró que los estudiantes realizaran prácticas de laboratorios sin estar acotado al tiempo de experimentación destinado en las clases presenciales.

Con la aplicación de la técnica ladov se midió el nivel de satisfacción de los usuarios mediante encuesta realizada a estudiantes del curso 20182019, obteniéndose alta satisfacción por los usuarios sobre la propuesta de SLR.

\section{Referencias}

Aguilar, I.; Heredia, J. A. (2013). Simuladores y laboratorios virtuales para ingeniería en 
computación. Revista Iberoamericana para la Investigación y el Desarrollo Educativo, 10.

Arántegui, J. (2011). Control de procesos. http:// cort.as/-QLzq

Badamasi, Y. A. (2014). The working principle of an arduino. Electronics, Computer And Computation (IcecCo), 11th International Conference, 1-4. https://doi.org/10.1109/ ICECCO.2014.6997578

Buitrago-Molina, J. T.; Carvajal-Guerrero, J. S.; Zapata-Castillo, C. (2014). Plataforma virtual para el mando local y remoto de un brazo robótico de apoyo para la educación en ingeniería. Tecno Lógicas, 17, 67-74. https://doi. org/10.22430/22565337.206

Cáceres, C. A.; Amaya, D. (2016). Desarrollo e interacción de un laboratorio virtual asistido y controlado por PLC. Entre Ciencia E Ingeniería, 10, 9-15.

Castillo, J.; Ginoris, O. (2005). Formación y desarrollo de los intereses profesionales pedagógicos en los estudiantes de primer año de la licenciatura en educación como inductores del aprendizaje autodidacto. Instituto Superior Pedagógico Juan Marinello.

Garay, M. (2015). Interfaces inteligentes en el aprendizaje de la modelación. Ingeniería Industrial, 36(2), 187-201.

González, J. (2013). Propuesta de algoritmo de clasificación genética. Revista Cubana de Ingeniería, 4(2), 37-42.

Jin-Hsien, W.; Jongyun, H. (2007). An approach to computing with words based on canonical characteristic values of linguistic labels fuzzy systems. leee Transactions On, 15(4), 593-604. https://doi.org/10.1109/ TFUZZ.2006.889844

Kuzmina, N. (1970). Metódicas investigativas de la actividad pedagógica. Moscú, Rusia: Editorial Leningrado.

López, A.; González, V. (2002). La técnica de ladov una aplicación para el estudio de la satisfacción de los alumnos por las clases de educación física. Revista Digital-Buenos Aires, 8(47).
Mar, O.; Gulín, J.; Santana, I.; Rozhnova, L. (2016). Sistema de laboratorios a distancia para la práctica de control automático. Revista Cubana de Ciencias Informáticas, 10(4), 171-183.

Mar, O.; Santana, I.; González, J.; Rozhnova, L. (2017). Competency assessment model for a virtual laboratory system and distance using fuzzy cognitive map. Investigación Operacional, 38(2), 170-178.

Matijevic, M.; Cvjetkovic, V. (2016). Overview of architectures with arduino boards as building blocks for data acquisition and control systems. 13th International Conference On Remote Engineering And Virtual Instrumentation (Rev), 56-63. https://doi.org/10.1109/ $\underline{\text { REV.2016.7444440 }}$

Hernández, L. (2017). Ingeniería en Automática realizó la defensa del Plan de Estudios E. https://www.uclv.edu.cu/ingenieria-automatica-realizo-la-defensa-del-plan-estudios-e/

Murthy, A.; Rao, N.; Ranganahalli, Y.; Shandilya, S.; Badarmanahalli, R. (2014). Design and construction of arduino-hacked variable gating distortion pedal. leee Access, 2, 1409-1417. https://doi.org/10.1109/ACCESS.2014.2374195

Ogata, K. (2010). Modern Control Engineering. Minnesota, EE. UU.: Editorial Engineering/ Computer Science.

Rubio, E.; Santana, I.; Esparza, V; Rohten, J. (2016). Remote Laboratories For Control Education: Experience At The Universidad Del Bío-Bío. leee International Conference On Automatica (Ica-Acca), 1-6. https://doi.org/10.1109/ ICA-ACCA.2016.7778444

Saenz, J.; Chacón, J.; de la Torre, L.; Visioli, A.; Dormido, S. (2015). Open and low-cost virtual and remote labs on control engineering. leee Access, 3, 805-814. https://doi.org/10.1109/ ACCESS.2015.2442613

Salas, M.; Cerón, C. (2014). Sistema web para evaluar las competencias mediante pruebas objetivas en educación superior. Revista Iberoamericana para la Investigación y el DesarroIlo Educativo, 12. 
Samuelsen, D. A. H.; Graven, O. H. (2016). Remote laboratories in engineering education - an overview of implementation and feasability. Laccei International Multi-Conference For Engineering, Education And Technology. $\quad$ http://dx.doi.org/10.18687/ LACCEI2016.1.1.050

Sancristobal-Ruiz, E.; Pesquera, A.; et al. (2014). Virtual and remote industrial laboratory: integration in learning management systems. IEEE Industrial Electronics Magazine, 8(4), 45-58.

Santana, I. (2012). Herramientas para la docencia en automática orientadas hacia la metodología (tesis doctoral). Departamento de Automática, Ingeniería Electrónica e Informática Industrial, Escuela Técnica Superior de Ingenieros Industriales, Madrid.
Santana, I.; Ferre, M.; Izaguirre, E.; Aracil, R.; Hernández, L. (2013). Remote laboratories for education and research purposes in automatic control systems industrial informatics. IEEE Transactions On, 9(1), 547-556. https://doi. org/10.1109/TII.2011.2182518

Sartorius C. A. R.; Hernández, L. S.; Aracil, R. (2005). Laboratorio a distancia para la prueba y evaluación de controladores a través de internet. Sba: Controle \& Automação Sociedade Brasileira De Automatica, 16, 84-92. https:// doi.org/10.1590/S0103-17592005000100010

Valencia, A. (2016). El futuro de la ingeniería. Revista Facultad de Ingeniería,19, 85-92.

Ziegler, J. G.; Nichols, N. B. (1942). Optimum settings for automatic controllers. Americana de Ingenieros Mecánicos (Asmet) Transactions, 64(11), 1-10. 